\title{
A Method for Calculating the Association Degrees between Concepts of Concept Networks
}

\author{
Shi-Jay Chen \\ Department of Information Management, National United University, Miaoli, China \\ Email: sjchen@nuu.edu.tw
}

How to cite this paper: Chen, S.-J. (2018) A Method for Calculating the Association Degrees between Concepts of Concept Networks. Journal of Computer and Communications, 6, 55-65. https://doi.org/10.4236/jcc.2018.65005

Received: March 26, 2018

Accepted: May 21, 2018

Published: May 24, 2018

Copyright $\odot 2018$ by author and Scientific Research Publishing Inc. This work is licensed under the Creative Commons Attribution International License (CC BY 4.0).

http://creativecommons.org/licenses/by/4.0/

\begin{abstract}
Depicting the associating degrees between two concepts and their relationships are major works for constructing a multi-relationship fuzzy concept network. This paper indicates some drawbacks of the existing methods of calculating associating degrees between concepts, and proposes a new method for overcoming these drawbacks. We also use some examples to compare the proposed method with the existing methods for calculating the associating degrees between two concepts in a multi-relationship fuzzy concept networks.
\end{abstract}

\section{Keywords}

Document Retrieval, Fuzzy Query Processing, Geometric-Mean Averaging Operators, Fuzzy Concept Networks

\section{Introduction}

Salton and Mcgill proposed information retrieval system based on the Boolean logic model [1]. Moreover, documents are retrieved only when they contain the index terms specified in the user's queries. However, this method may be neglect some relevant documents that do not contain the index terms specified in user's queries. Therefore, many researchers proposed intelligent information retrieval systems to retrieval documents intelligently by incorporating knowledge bases into the systems [2]-[13]. In [13], Lucarella et al. presented the fuzzy concept networks for information retrieval based on fuzzy set theory [14]. The concept network can depict the relationships between concepts which are defined as index terms [15] or classes of documents [11] in a specific domain.

In [9], Horng et al. proposed the method to automatically construct multi-relationship fuzzy concept networks for fuzzy information retrieval. In mul- 
ti-relationship, there are four kinds of relationship to describe possible semantic relationships between concepts, such as fuzzy positive association relationship, fuzzy negative associating relationship, fuzzy generalization relationship and fuzzy specialization relationship [10]. The users of the fuzzy information retrieval system based on multi-relationship concept networks can submit a fuzzy query in which a search context is involved to provide the user's perspective on the fuzzy relationships between concepts. Documents are retrieved if they contain concepts that have a specified fuzzy relationship with the concepts contained in the user's query when concerning the search context. Thus, depicting the associating degrees between two concepts and their relationships are important for constructing a multi-relationship fuzzy concept network.

The rest of this study is organized as follows. Section 2 briefly reviews the concept of geometric mean, the fuzzy concept network [13] and the muti-relationship fuzzy concept network [10]. Section 3 reviews the existing methods of associating degrees between concepts for automatically constructing multi-relationship associating fuzzy concept networks, and indicates some drawbacks of existing methods for calculating associating degrees between concepts. Section 4 presents a new method for calculating associating degrees between concepts, and uses some examples to compare the proposed method with the existing methods. Conclusions are finally drawn in Section 5.

\section{Preliminary}

In [9], the geometric mean of positive number $a_{1}, a_{2}, \cdots, a_{n}$ is defined as

$$
\sqrt[n]{\prod_{i=1}^{n} a_{i}}=\sqrt[n]{a_{1} \times a_{2} \times \cdots \times a_{n}},
$$

where $1 \leq i \leq n$. The geometric mean is well defined for sets of positive numbers, and is useful to deal with fuzzy aggregating problem and fuzzy decision-making problem.

\subsection{Fuzzy Concept Networks}

Lucarella et al. have proposed the fuzzy concept networks for fuzzy information retrieval [13]. A fuzzy concept network includes nodes and directed links. Each node represents a concept or document. Each directed link connects two concepts or directs from one concept $c_{i}$ to one document $d_{i}$, and each directed link is associated with a degree $\mu$, where $\mu \in[0,1]$, indicating the degree of strength of the relationship between two concepts or the degree of strength that a document contains a concept. Figure 1 shows a fuzzy concept network, where $c_{1}, c_{2}, \cdots$ and $c_{7}$ are concepts, and $d_{1}, d_{2}, d_{3}$ and $d_{4}$ are documents. A link in the fuzzy concept network is defined as:

$$
l=\{\langle c, r\rangle, u(c, r) \mid c \in C \text { and } r \in C\},
$$

where $C$ represents the set of concepts, $u$ is the membership function, $u: C \times C \rightarrow[0,1]$, which represents that the concept $c$ and concept $r$ are connected 


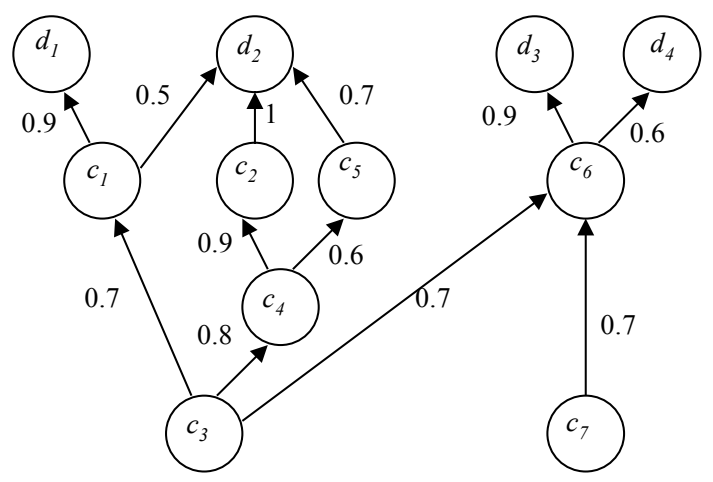

Figure 1. A fuzzy concept network.

by the link $l$, and their relevant is $u(c, r)$, where $u(c, r) \in[0,1]$.

In the relevant value between concept $c$ and concept $r$ is $u(c, r)$, and the relevant value between concept $r$ and concept $s$ is $u(r, s)$. Then based on the transitivity of link relationship, we can obtain the relevant value between concept $c$ and concept $s$ by the following expression:

$$
u(c, s)=\min (u(c, r), u(c, s)) .
$$

Similarly, if $u\left(c_{1}, c_{2}\right), u\left(c_{2}, c_{3}\right), \cdots, u\left(c_{n-1}, c_{n}\right)$ are known, then based on the transitivity of relationship, we can obtain the relevant value between concept $c_{1}$ and concept $c_{n}$ by the following expression:

$$
u\left(c_{1}, c_{n}\right)=\min \left(u\left(c_{1}, c_{2}\right), u\left(c_{2}, c_{3}\right), \cdots, u\left(c_{n-1}, c_{n}\right)\right) .
$$

\subsection{Multi-Relationship Fuzzy Concept Networks}

Kracker proposed the multi-relationship fuzzy concept network [10]. The concepts of multi-relationship fuzzy concepts are similar to the concepts of semantic networks [9] for expressing different types of relationship between keywords. Four types of relationship can be described the possible relationship between concepts in a multi-relationship fuzzy concept network as follows:

1) Positive association: It relates concepts with a fuzzy similar meaning (e.g. person-individual) in some contexts.

2) Negative association: It relates concepts with fuzzy complementary relationship (e.g. male-female), fuzzy incompatible relationship (e.g. unemployed-freelance) or fuzzy antonymous relationship (e.g. small-large) in some contexts.

3) Generalization: A concept regarded as a fuzzy generalization of another concept if it includes that concept in an analytic or partitive sense (e.g. person-student).

4) Specialization: It is the inverse of fuzzy generalization.

Let $C$ be a set of concepts in a multi-relationship fuzzy concept network. The fuzzy relationships between concepts are defined as follows [10].

1) Fuzzy positive associating $P$ is a fuzzy relation, $P: C \times C \rightarrow[0,1]$, which is reflexive, symmetric, and $\max ^{-}{ }^{*}$-transitive. 
2) Fuzzy negative association $N$ is a fuzzy relation, $N: C \times C \rightarrow[0,1]$, which is anti-reflexive, symmetric, and max-*-nontransitive.

3) Fuzzy generalization $G$ is a fuzzy relation, $G: C \times C \rightarrow[0,1]$, which is anti-reflexive, anti-symmetric, and max- ${ }^{*}$-transitive.

4) Fuzzy specialization $S$ is a fuzzy relation, $S: C \times C \rightarrow[0,1]$, which is anti-reflexive, anti-symmetric, and max-*-transitive.

A multi-relationship fuzzy concept network is denoted as $\operatorname{MRFCN}(E, L)$, where $E$ is a set of nodes, and where represents a concept or a document as in Figure 2. $L$ is a set of directed edges between nodes. If $l \in L$, then the directed edge $\mathrm{l}$ has following two formats:

1) $c_{i} \stackrel{\left(\left\langle\mu_{P}, P\right\rangle,\left\langle\mu_{N}, N\right\rangle,\left\langle\mu_{G}, G\right\rangle,\left\langle\mu_{S}, S\right\rangle\right)}{\longrightarrow} c_{j}$, means that the directed edge $I$ connect $c_{i}$ to $c_{j}$ with a four-tuple $\left(\left\langle\mu_{P}, P\right\rangle,\left\langle\mu_{N}, N\right\rangle,\left\langle\mu_{G}, G\right\rangle,\left\langle\mu_{S}, S\right\rangle\right)$, where $\mu_{P} \in[0,1]$, $\mu_{N} \in[0,1], \mu_{G} \in[0,1]$ and $\mu_{S} \in[0,1]$.

2) $c_{i} \stackrel{\mu}{\longrightarrow} d_{j}$, means that document $d_{j}$ has concept $c_{i}$ with the degree of strength, where $\mu \in[0,1]$.

Figure 2 shows a multi-relationship fuzzy concept network, where $c_{1}, c_{2}, \cdots, c_{7}$ are concepts; $d_{1}, d_{2}, d_{3}$ and $d_{4}$ are documents.

Furthermore, Horng et al. proposed an algorithm with eight steps to construct multi-relationship fuzzy concept networks automatically [9].

\section{Analysis of the Existing Methods for Calculating the Relationships and the Associating Degrees between Concepts}

In [9], Horng et al. pointed out that calculating the relationships and the associating degrees between concepts is an important part of constructing a multi-relationship fuzzy concept network. They decided fuzzy relationship between two concepts by following six cases. Assume the concept $c_{i}$ and the concept $c_{j}$ be any two arbitrary concepts in the concept set $C$, the discussions of the six cases are shown as follows.

Case 1: If concept $c_{i}$ and concept $c_{j}$ contain different words, then they are not related.

Case 2: If concept $c_{i}$ and concept $c_{j}$ contain almost the same words, but the weighs of the words in concept $c_{i}$ are larger than those in concept $c_{p}$ then concept $c_{i}$ is said to dominate concept $c_{j}$ and should be more general than concept $c_{j}$

Case 3: If concept $c_{i}$ and concept $c_{j}$ contain almost the same words, but the weights of the words in concept $c_{i}$ are smaller than those in concept $c_{p}$ then concept $c_{i}$ is said to be dominated by con concept $c_{j}$ and should be more specific than concept $c_{j}$

Case 4: If most words contained in concept $c_{j}$ are also contained in concept $c_{p}$ but many words contained in concept $c_{i}$ are not contained in concept $c_{p}$ then concept $c_{i}$ concerns more aspects than concept $c_{j}$ and should be more general than concept $c_{j}$ 


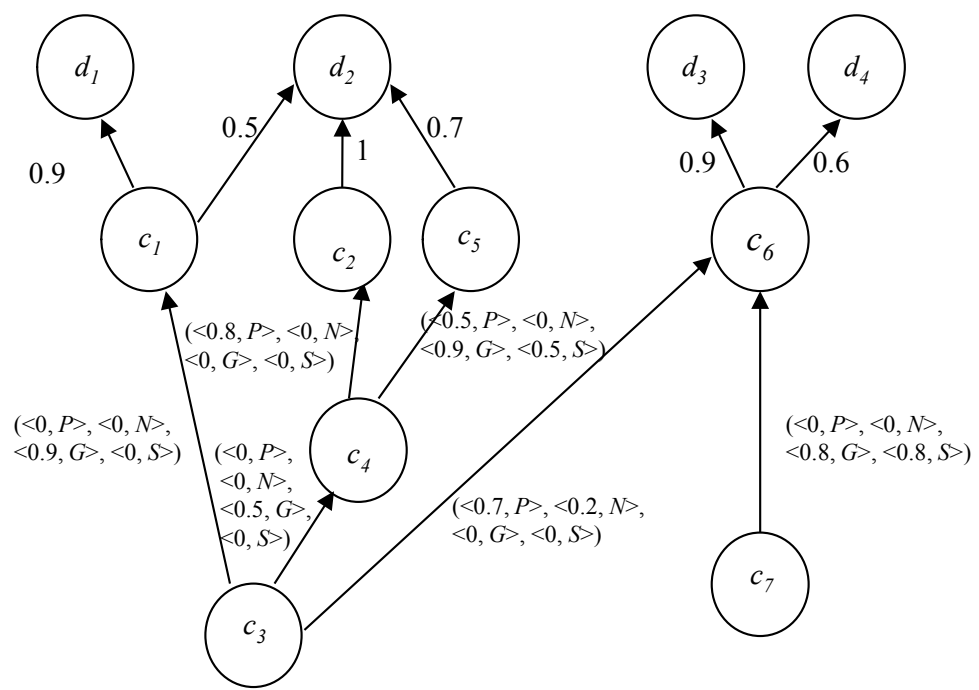

Figure 2. A multi-relationship fuzzy concept network.

Case 5: If most words contained in concept $c_{i}$ are also contained in concept $c_{p}$, but many words contained in concept $c_{j}$ are not contained $c_{i}$, then concept $c_{i}$ concerns fewer aspect than concept $c_{j}$ and should be more specific than concept $c_{j}$

Case 6: If concept $c_{i}$ and concept $c_{j}$ contain almost the same words, and the weight of the words are similar in both concepts, then these two concepts should be similar to each other and have a fuzzy positive associating relationship.

Young proposed a method for calculating the associating degree between concepts [16]. The proposed method uses a mapping function $M$ to represent each concept by showing its corresponding fuzzy subset in the word set WS. The mapping function $M$ shown as follows:

$$
M\left(c_{i}\right)=w_{i 1} / t_{1}+w_{i 2} / t_{2}+\cdots+w_{i h} / t_{h},
$$

where $M: C \rightarrow[0,1]^{W}, W_{i 1}$ is the weight of word $t_{j}$ in concept $c_{i}$, and $h$ is the number of words in the word set WS. Then, calculating the associating degree between concepts denoted $G\left(c_{i}, c_{j}\right)$ and equal to the degree of subsethood of $M\left(c_{i}\right)$ in $M\left(c_{j}\right)$. A method to calculate $G\left(c_{i}, c_{j}\right)$ is shown as follows:

$$
G\left(c_{i}, c_{j}\right)= \begin{cases}\left(\frac{\left|M\left(c_{i}\right) \cap M\left(c_{i}\right)\right|}{\left|M\left(c_{i}\right)\right|}\right)=\left(\frac{\sum_{k=1}^{h} \min \left(w_{k i}, w_{k j}\right)}{\sum_{k=1}^{h} w_{k i}}\right), & \text { if } M\left(c_{j}\right) \neq \phi \\ 1, & \text { if } M\left(c_{i}\right) \neq \phi\end{cases}
$$

where $w_{k i}$ is the weight of word $t_{k}$ in concept $c_{i}, \mathrm{w}_{k i}$ is the weight of word $t_{k}$ in concept $c_{p} W C\left(c_{i}\right)$ is the number of words contained on concept $c_{i}, W C\left(c_{j}\right)$ is the number of words contained in concepts $c_{p}$, and $h$ is the number of words in the word set WS. According to Subsection 2.2, we can understand that fuzzy specialization relationship is the inverse of the fuzzy generalization relationship. Thus

$$
S\left(c_{i}, c_{j}\right)=G\left(c_{i}, c_{j}\right) .
$$


Moreover, based on Subsection 2.1, the degree of fuzzy positive association relationship between concept $c_{i}$ and concept $c_{p}$, denoted as $P\left(c_{i}, c_{j}\right)$, is calculated as follows:

$$
P\left(c_{i}, c_{j}\right)=\min \left(G\left(c_{i}, c_{j}\right), S\left(c_{i}, c_{j}\right)\right) .
$$

However, Horng et al. [9] founded that Young's method cannot effectively reveal the generality of concept $c_{j}$ over concept $c_{i}$, such as the following example [9].

Example 3.1: Assume that there are five words $t_{1}, t_{2}, \ldots$, and $t_{5}$ in the word set $W S$ and assume that the corresponding fuzzy subset $M\left(c_{i}\right)$ and $M\left(c_{j}\right)$ of concept $c_{i}$ and concept $c_{j}$ in the word set $W S$ are shown as follows:

$$
\begin{gathered}
M\left(c_{i}\right)=0.3 / t_{1}+0.3 / t_{2}+0.4 / t_{3}+0.4 / t_{4}+0.3 / t_{5}, \\
M\left(c_{j}\right)=0.8 / t_{2}+0.9 / t_{3} .
\end{gathered}
$$

According to Case 4 of the above six cases for deciding fuzzy relationship between concepts, concept $c_{i}$ should be more general than the concept $c_{j}$ because concept $c_{i}$ contains all the words contained in concept $c_{j}$ (i.e., the words $t_{2}, t_{3}$ ). However, Young's method yield the same associating degrees $G\left(c_{i}, c_{j}\right)$ and $G\left(c_{j}\right.$ $\left.c_{i}\right)$ as follows:

$$
\begin{gathered}
G\left(c_{i}, c_{j}\right)=\frac{0.3+0.4}{0.3+0.3+0.4+0.4+0.3}=0.41, \\
G\left(c_{j}, c_{i}\right)=\frac{0.3+0.4}{3.8+0.9}=0.41 .
\end{gathered}
$$

According to the above results, we cann't know which concept is more general than the other one.

Therefore, Horng et al. [9] proposed the formula (6) to overcome this drawback.

$$
G\left(c_{i}, c_{j}\right)= \begin{cases}\left(\frac{\sum_{k=1}^{h} \min \left(w_{k i}, w_{k j}\right)}{\sum_{k=1}^{h} w_{k i}}\right)^{\frac{W C\left(c_{i}\right)}{\max \left(W C\left(c_{i}\right), W C\left(c_{j}\right)\right)}} & \\ 1, & \text { if } M\left(c_{j}\right) \neq \phi\end{cases}
$$

where $w_{k i}$ is the weight of word $t_{k}$ in concept $c_{i}, w_{k j}$ is the weight of word $t_{k}$ in concept $c_{p} W C\left(c_{i}\right)$ is the number of words contained on concept $c_{p}, W C\left(c_{j}\right)$ is the number of words contained in concepts $c_{p}$ and $h$ is the number of words in the word set WS. The proposed method can overcome the drawback of Young's method.

However, we also found the formula (6) proposed by Horng et al. still has some drawbacks for dealing with associating degrees between concepts (i.e., the result is not fitting for one of the above six cases). In the following, we use some examples to illustrate these drawbacks.

Example 3.2: Assume there are four words $t_{1}, t_{2}, t_{3}$ and $t_{4}$ in the word set WS, and assume that the corresponding fuzzy subset $M\left(c_{i}\right)$ and $M\left(c_{j}\right)$ of concept $c_{i}$ 
and concept $c_{j}$ in the word set $W S$ are shown as follows:

$$
\begin{gathered}
M\left(c_{i}\right)=0.3 / t_{1}+0.1 / t_{2}+0.2 / t_{3}+0.8 / t_{4}, \\
M\left(c_{j}\right)=0.4 / t_{1}+0.3 / t_{2}+0.8 / t_{3} .
\end{gathered}
$$

According to Case 2 of the above six cases for deciding fuzzy relationship between concepts, concept $c_{j}$ should be more general than concept $c_{i}$ because concept $c_{i}$ and concept $c_{j}$ contained almost the same words (i.e., the words $t_{1}, t_{2}$, and $t_{3}$ ), but all the weights of the words in concept $c_{j}$ also contained in concept $c_{i}$ are larger than concept $c_{i}$. Based on Horng et al.'s method, we calculate the $G\left(c_{i}, c_{j}\right)$ and $G\left(c_{j}, c_{i}\right)$, respectively, as follows:

$$
\begin{gathered}
G\left(c_{i}, c_{j}\right)=\left(\frac{0.3+0.1+0.2}{0.3+0.1+0.2+0.8}\right)^{\frac{4}{4}}=0.42, \\
G\left(c_{j}, c_{i}\right)=\left(\frac{0.3+0.1+0.2}{0.4+0.3+0.8}\right)^{\frac{3}{4}}=0.5 .
\end{gathered}
$$

Since $G\left(c_{p} c_{i}\right)$ is larger than $G\left(c_{p}, c_{j}\right)$, we can see that concept $c_{i}$ is more general than concept $c_{j}$. However, the relationship between the two concepts $c_{i}$ and $c_{j}$ does not coincide with human intuition for violating Case 2.

Example 3.3: Assume that there are six words $t_{1}, t_{2}, \cdots, t_{6}$ in the word set $W S$, and assume that the corresponding fuzzy subset $M\left(c_{i}\right)$ and $M\left(c_{j}\right)$ of concept $c_{i}$ and concept $c_{j}$ in the word set $W S$ are shown as follows:

$$
\begin{gathered}
M\left(c_{i}\right)=0.6 / t_{1}+0.3 / t_{2}+0.4 / t_{3}+0.7 / t_{4}+0.6 / t_{5}+1 / t_{6}, \\
M\left(c_{j}\right)=0.8 / t_{1}+0.5 / t_{2}+0.7 / t_{3}+1 / t_{4}+0.8 / t_{5} .
\end{gathered}
$$

According to Case 3 of the above six cases for deciding fuzzy relationship between concepts, concept $c_{j}$ is general than $c_{i}$ because the two concepts $c_{i}$ and $c_{j}$ contained almost the same words (i.e., the word $t_{1}, t_{2}, t_{3}, t_{4}$ and $t_{5}$ ), but all the weights of the words in concept $c_{j}$ also contained in concept $c_{i}$ are larger than concept $c_{i}$. Based on Horng et al.'s method, we calculate the $G\left(c_{i p} c_{j}\right)$ and $G\left(c_{p}, c_{i}\right)$, respectively, as follows:

$$
\begin{gathered}
G\left(c_{i}, c_{j}\right)=\left(\frac{0.6+0.3+0.4+0.7+0.6}{0.6+0.3+0.4+0.7+0.6+1}\right)^{\frac{6}{6}}=0.6767, \\
G\left(c_{j}, c_{i}\right)=\left(\frac{0.6+0.3+0.4+0.7+0.6}{0.8+0.5+0.7+1+0.8}\right)^{\frac{5}{6}}=0.6842 .
\end{gathered}
$$

Since $G\left(c_{j}, c_{i}\right)$ is larger than $G\left(c_{i}, c_{j}\right)$, we can see that concept $c_{i}$ is more general than concept $c_{j}$. However, the relationship between the two concepts $c_{i}$ and $c_{j}$ is not fitting for human intuition because of violating Case 3.

Example 3.4: Assume that there are seven words $t_{1}, t_{2}, \cdots, t_{7}$ in the word set $W S$, and assume that the corresponding fuzzy subset $M\left(c_{i}\right)$ and $M\left(c_{j}\right)$ of concept $c_{i}$ and concept $c_{j}$ in the word set $W S$ are shown as follows:

$$
M\left(c_{i}\right)=1 / t_{1}+0.8 / t_{2}+0.9 / t_{3}
$$




$$
M\left(c_{j}\right)=0.2 / t_{1}+0.1 / t_{2}+0.2 / t_{3}+0.1 / t_{4}+0.2 / t_{5}+0.1 / t_{6}+0.1 / t_{7} .
$$

According to Case 4 of the above six cases for deciding fuzzy relationship between concepts, concept $c_{j}$ should be more general than the concept $c_{i}$ because concept $c_{j}$ contains all the words contained in concept $c_{p}$ (i.e., the words $t_{1}, t_{2}$ and $t_{3}$ ), but the words $t_{4}, t_{5}, t_{6}$ and $t_{7}$ contained in concept $c_{j}$ are not contained in concept $c_{i}$. Based on Horng et al.'s method, we calculate the $G\left(c_{p}, c_{j}\right)$ and $G\left(c_{p}, c_{i}\right)$, respectively, as follows:

$$
\begin{gathered}
G\left(c_{i}, c_{j}\right)=\left(\frac{0.2+0.1+0.2}{1+0.8+0.9}\right)^{\frac{3}{7}}=0.485 \\
G\left(c_{j}, c_{i}\right)=\left(\frac{0.2+0.1+0.2}{0.2+0.1+0.2+0.1+0.2+0.1+0.1}\right)^{\frac{4}{4}}=0.5 .
\end{gathered}
$$

Since $G\left(c_{i}, c_{j}\right)$ is larger than $G\left(c_{p} c_{i}\right)$, we can see that concept $c_{i}$ is more general than concept $c_{i}$. However, the relationship between the two concepts $c_{i}$ and $c_{j}$ does not coincide with human intuition because of violating Case 4 .

According to the above discussion, we found that formula (5) proposed by Horng et al. has some drawbacks for calculating the degrees between concepts. In order to obtain more accurate associating degrees between concepts for automatically constructing multi-relationship fuzzy concept networks, to develop a new method for calculating associating degrees between concepts is necessary.

\section{A New Method for Calculating Associating Degrees between Two Concepts}

In this section, we present a new method for calculating associating degrees between concepts based on geometric mean operator. The new method for calculating associating degrees between concepts shown as follows:

$$
G\left(c_{i}, c_{j}\right)= \begin{cases}\frac{\sum_{k=1}^{h} \sqrt{w_{k i} \times w_{k j}}}{2 \times \sum_{k=1}^{k} w_{k i}}+(0.5)^{1+2 \times \operatorname{ROUND}\left(\frac{W C\left(c_{i}\right)}{\max \left(W C\left(c_{i}\right), W C\left(c_{j}\right)\right)}\right),}, & \text { if } M\left(c_{j}\right) \neq \phi \\ 1, & \text { if } M\left(c_{i}\right) \neq \phi\end{cases}
$$

where $w_{k i}$ is the weight of word $t_{k}$ in concept $c_{i}, w_{k j}$ is the weight of word $t_{k}$ in concept $c_{p} W C\left(c_{i}\right)$ is the number of words contained in concept $c_{p} W C\left(c_{j}\right)$ is the number of the words contained in concept $c_{p}$ and $h$ is the number of words in the word set WS. ROUND(.) is a round off function, e.g. $\operatorname{ROUND}(0.4)=0$ and $\operatorname{ROUND}(0.6)=1$. The main idea of the proposed method is to include the rate of words contained in concept. We have found that if we increase the importance of the rate of words contained in concept while the rate above 0.5 , we can get the appropriate association degrees between concepts. Regarding the weight values of the word in concept, some references use the number of words in the document to calculate the weight [1]. Young's method and Horng et al.'s method all use min operator to obtain the associating degree $\left.G\left(c_{i}\right) c_{j}\right)$ for constructing a fuzzy concept network. However, Kim et al. pointed out that the min and 
max operators have the "single operand dependent" property, which decrease retrieval effectiveness [15]. Thus, our method is based on geometric mean for calculating the associating degrees between concepts.

In the following, we use the examples discussed in Section 3 to compare the proposed method with existing methods.

1) If we use formula (7) to deal with Example 3.1, we can calculate the two associating degrees $G\left(c_{p}, c_{j}\right)$ and $G\left(c_{p} c_{i}\right)$, respectively, as follows:

$$
\begin{gathered}
G\left(c_{i}, c_{j}\right)=\frac{\sqrt{0.3 \times 0.8}+\sqrt{0.4 \times 0.9}}{2 \times(0.3+0.3+0.4+0.4+0.3)}+(0.5)^{1+2 \times \operatorname{ROUND}\left(\frac{5}{5}\right)}=0.44556 \\
G\left(c_{j}, c_{i}\right)=\frac{\sqrt{0.3 \times 0.8}+\sqrt{0.4 \times 0.9}}{2 \times(0.8+0.9)}+(0.5)^{1+2 \times \operatorname{ROUND}\left(\frac{2}{5}\right)}=0.82056 .
\end{gathered}
$$

Since $G\left(c_{p} c_{i}\right)$ is larger than $G\left(c_{p}, c_{j}\right)$, we can see that concept $c_{i}$ is more general than concept $c_{p}$ and it coincides with the intuition of the human being.

2) If we use formula (7) to deal with Example 3.2, we can calculate the two associating degrees $G\left(c_{p}, c_{j}\right)$ and $G\left(c_{p} c_{i}\right)$, respectively, as follows:

$$
\begin{aligned}
& G\left(c_{i}, c_{j}\right)=\frac{\sqrt{0.3 \times 0.4}+\sqrt{0.1 \times 0.3} \times \sqrt{0.2 \times 0.8}}{2 \times(0.3+0.1+0.2+0.8)}+(0.5)^{1+2 \times \operatorname{ROUND}\left(\frac{4}{4}\right)}=0.45343, \\
& G\left(c_{j}, c_{i}\right)=\frac{\sqrt{0.3 \times 0.4}+\sqrt{0.1 \times 0.3} \times \sqrt{0.2 \times 0.8}}{2 \times(0.4+0.3+0.8)}+(0.5)^{1+2 \times \operatorname{ROUND}\left(\frac{3}{4}\right)}=0.43154 .
\end{aligned}
$$

Since $G\left(c_{p} c_{i}\right)$ is larger than $G\left(c_{p} c_{j}\right)$, we can see that concept $c_{i}$ is more general than concept $c_{p}$ and it coincides with the intuition of the human being for observing Case 2.

3) If we use formula (7) to deal with Example 3.3, we can calculate the two associating degrees $G\left(c_{p} c_{j}\right)$ and $G\left(c_{p} c_{i}\right)$, respectively, as follows:

$$
\begin{aligned}
G\left(c_{i}, c_{j}\right)= & \frac{\sqrt{0.6 \times 0.8}+\sqrt{0.3 \times 0.5}+\sqrt{0.4 \times 0.7}+\sqrt{0.7 \times 0.1}+\sqrt{0.6 \times 0.8}}{2 \times(0.6+0.3+0.4+0.7+0.6+1)} \\
& +(0.5)^{1+2 \times \operatorname{ROUND}\left(\frac{6}{6}\right)} \\
= & 0.5609 \\
G\left(c_{j}, c_{i}\right)= & \frac{\sqrt{0.6 \times 0.8}+\sqrt{0.3 \times 0.5}+\sqrt{0.4 \times 0.7}+\sqrt{0.7 \times 1}+\sqrt{0.6 \times 0.8}}{2 \times(0.8+0.5+0.7+1+0.8)} \\
& +(0.5)^{1+2 \times \operatorname{Round}\left(\frac{5}{6}\right)} \\
= & 0.5380
\end{aligned}
$$

Since $G\left(c_{p}, c_{j}\right)$ is larger than $G\left(c_{p} c_{i}\right)$, we can see that concept $c_{j}$ is more general than concept $c_{p}$ and it coincides with the intuition of the human being for observing Case 3.

4) If we use formula (7) to deal with Example 3.4, we can calculate the two associating degrees $G\left(c_{p}, c_{j}\right)$ and $G\left(c_{p} c_{i}\right)$, respectively, as follows:

$$
G\left(c_{i}, c_{j}\right)=\frac{\sqrt{0.1 \times 0.2}+\sqrt{0.8 \times 0.1} \times \sqrt{0.9 \times 0.2}}{2 \times(0.1+0.8+0.9)}+(0.5)^{1+2 \times \operatorname{ROUND}\left(\frac{3}{7}\right)}=0.71376 \text {, }
$$




$$
\begin{aligned}
G\left(c_{j}, c_{i}\right) & =\frac{\sqrt{0.3 \times 0.4}+\sqrt{0.1 \times 0.3} \times \sqrt{0.2 \times 0.8}}{2 \times(0.2+0.1+0.2+0.1+0.2+0.1+0.1)}+(0.5)^{1+2 \times \operatorname{ROUND}\left(\frac{7}{7}\right)} . \\
& =0.70216
\end{aligned}
$$

Since $G\left(c_{i}, c_{j}\right)$ is larger than $G\left(c_{j}, c_{i}\right)$, we can see that concept $c_{j}$ is more general than concept $c_{i}$, and it coincides with the intuition of the human being for observing Case 4.

From the previous discussions, we can obtain the proposed method is useful than the two existing methods proposed by Young and Horng et al. respectively for calculating the associating degrees between two concepts for deciding their relationship in a multi-relationship fuzzy concept network.

\section{Conclusion}

In this paper, we firstly pointed out some drawbacks of the existing methods for calculating the associating degree between two concepts, and presented a method based on geometric mean operator for overcoming these drawbacks. We used some examples to compare the proposed method with the existing methods. The proposed method is more useful than the existing methods to calculate the associating degrees between two concepts for constructing their relationship in a multi-relationship fuzzy concept networks for document retrieval.

\section{Acknowledgements}

This work was supported in part by the Ministry of Science and Technology, Republic of China, under Grant 104-2410-H-239-007.

\section{References}

[1] Salton, G. and Mcgill, M.J. (1983) Introduction to Modern Information Retrieval. McGraw-Hill Education, New York.

[2] Bezdek, J.C., Biswas, G. and Huang, L.Y. (1986) Transitive Closures of Fuzzy Thesauri for Information-Retrieval System. International Journal of Man-Machine Studies, 25, 343-356. https://doi.org/10.1016/S0020-7373(86)80065-7

[3] Bhatia, S.K. and Deogun, J.S. (1998) Conceptual Clustering on Information Retrieval. IEEE Transactions on Systems, Man, and Cybernetics-Part B, Cybernetics, 28, 427-435. https://doi.org/10.1109/3477.678640

[4] Chang, C.S. and Chen, A.L.P. (1998) Supporting Conceptual and Neighborhood Quiries on the World Wide Web. IEEE Transactions on Systems, Man, and Cybernetics-Part B, Cybernetics, 28, 300-308. https://doi.org/10.1109/5326.669578

[5] Chen, C.L.P. and Lu, Y. (1997) FUZZY: A Fuzzy-Based Concept Information System That Integrates Human Categorization and Numerical Clustering. IEEE Transactions on Systems, Man, and Cybernetics-Part B, Cybernetics, 27, 79-94. https://doi.org/10.1109/3477.552187

[6] Chen, S.M. and Horng, Y.J. (1999) Fuzzy Query Processing for Document Retrieval Based on Extended Fuzzy Concept Networks. IEEE Transactions on Systems, Man, and Cybernetics-Part B, Cybernetics, 29, 96-104.

[7] Chen, S.M., Horng, Y.J. and Lee, C.H. (2000) Fuzzy Information Retrieval Method Based on Multi-Relationship Fuzzy Concept Networks. Proceedings of the 2000 In- 
ternational Computer Symposium: Workshop on Artificial Intelligence, Chiayi, 6-8 December 2000, 79-86.

[8] Chen, S.M., and Wang, J.Y. (1995) Document Retrieval Using Knowledge-Based Fuzzy Information Retrieval Techniques. IEEE Transactions on Systems, Man, and Cybernetics, 25, 793-803.

[9] Horng, Y.J., Chen, S.M. and Lee, C.H. (2003) Automatically Constructing Multi-Relationship Fuzzy Concept Networks for Document Retrieval. Applied Artificial Intelligence, 17, 303-328. https://doi.org/10.1080/713827141

[10] Kracker, M. (1992) A Fuzzy Concept Network Model and Its Applications. Proceedings of the First IEEE International Conference on Fuzzy Systems, San Diego, 8-12 March 1992, 761-768. https://doi.org/10.1109/FUZZY.1992.258752

[11] Liang, T. and Chang, C.C., (1999) Chinese Textual Retrieval Based on Fuzzy Concept Networks. Proceedings of National Computer Symposium, Tamsui, 20-22 December1999, 61-67.

[12] Lin, C.C., Tseng, S.Y. and Chen, P.M (1999) A Fuzzy Document Retrieval System Based on Concept Networks and Cluster Analysis. Soochow Journal of Economics and Business, 25, 39-60.

[13] Lucarella, D. and Morara, R. (1991) FIRST: Fuzzy Information Retrieval System. Journal of Information Science, 17, 81-91. https://doi.org/10.1177/016555159101700202

[14] Zadeh, L.A. (1965) Fuzzy Sets. Information and Control, 8, 338-353. https://doi.org/10.1016/S0019-9958(65)90241-X

[15] Kim, K.J. and Cho, S.B. (2001) A Personalized Web Search Engine Using Fuzzy Concept Network with Link Structure. Proceedings of the Joint 9 th IFSA Congress and 20 th NAFIPS International Conference, Vancouver, 25-28 July 2001, 81-86.

[16] Young, V.R. (1996) Fuzzy Subsethood. Fuzzy Sets and Systems, 77, 371-384. https://doi.org/10.1016/0165-0114(95)00045-3 